\title{
THE GROUP OF HOLOEDRIC TRANSFORMATION INTO ITSELF OF A GIVEN GROUP.
}

\author{
Presented to the American Mathematical Society at the Meeting of \\ November 24, 1894.
}

BY PROFESSOR E. HASTINGS MOORE.

$\S 1$.

Every group $G_{n}$ of order $n$ determines a substitution-group $\Gamma^{n-1}$ of degree $n-1$.

Given an (abstract) group $G_{n}^{\gamma}$ of order $n$, with the elements $s_{1}=$ identity, $s_{2}^{\prime}, \ldots s_{n}$. The group $G_{n}$ is holoedrically isomorphic with itself if a 1-1 correspondence*

$$
\left(s_{1}, s_{2}, \ldots s_{n}\right) \sim\left(s_{\iota_{1}}, s_{\iota_{2}}, \ldots s_{\iota n}\right)
$$

can be set up amongst its elements of such a sort that whenever in $G_{n}$ we have $s_{f} s_{g}=s_{h}$ we have also $s_{t_{f}} s_{\iota_{g}}=s_{\iota_{h}}$. Obviously corresponding elements must have the same period.

Attributing an order to the correspondence (1) we have the substitution

$$
\sigma=\left(\begin{array}{lll}
s_{1}, & s_{2}, \ldots & s_{n} \\
s_{\iota_{1}}, & s_{\iota_{2}}, \ldots & s_{\iota_{n}}
\end{array}\right)
$$

which holoedrically transforms the group $G_{n}$ into itself, that is, it transforms the system of product relations

$$
s_{f} s_{g}=s_{h}
$$

into itself. The totality of such substitutions $\sigma$ constitutes a substitution-group $\Gamma^{n}$ of degree $n$, which I call the group of holoedric transformation into itself of the original abstract group $G_{n}$ of order $n$.

If we transform the elements of the group $G_{n}$ through any particular element of $G_{n}$ we obtain such an holoedric transformation of $G_{n}$ into itself. The totality of such transformations constitutes a sub-group of $\Gamma_{n}$.

The group $\Gamma^{n}$ on the $n$ elements $s_{1}, s_{2}, \ldots s_{n}$ is certainly intransitive, since elements of the same period in $G_{n}$ are permuted

\footnotetext{
* This correspondence may always be established by setting properly choseu generators in 1-1 correspondence.
} 
amongst themselves by the substitutions $\sigma$ of $\Gamma^{n}$. $\Gamma^{n}$ is isomorphic with various substitution-groups $\Gamma_{\left(p_{\iota}\right)}^{n_{\iota}}$, where a particular $\Gamma_{\left(p_{\iota}\right)}^{n_{\iota}}$ is a substitution-group on the, say, $n_{\iota}$ elements of period $p_{\iota}$. It will on occasion happen that $\Gamma^{n}, \Gamma_{\left(p_{\iota}\right)}^{n_{\iota}}$ are holoedrically isomorphic, in which case the abstract-group-properties of the $\Gamma^{n}$ may perhaps be more conveniently studied as they appear in the $\Gamma_{\left(p_{\iota}\right)^{\circ}}^{n_{\iota}}$. It will always be desirable to replace the $\Gamma^{n}$ by the $\Gamma^{n-1}$ on the $n-1$ elements $s_{2}, s_{3}, \ldots s_{n}$, since the identity-element $s$, is invariant.

Intending to discuss the general question somewhat further in a subsequent note, I proceed now to consider an instructive example.

\section{$\S 2$.}

The group $\Gamma_{168}^{7}$ of holoedric transformation into itself of the Abelian group $G_{8}$ whose elements are the identity and seven commutative elements of period two.

The Abelian or commutative group $G_{8}$ has the utmost regularity of internal structure. We define it by the generating elements $a, b, c$, with the generating relations

$$
a^{2}=b^{2}=c^{2}=1, \quad a b=b a, \quad a c=c a, \quad b c=c b,
$$

where 1 is the identity. The elements may have the following notation:

$$
\begin{aligned}
& a \quad b c=d \\
& \begin{array}{cll}
1 & c a=e & a b c=g \\
c & a b=f
\end{array}
\end{aligned}
$$

Clearly, if $k \neq l$ and $k l=m$, the four elements, $1, k, l, m$ form a four-group. Three such elements $k, l, m$ (whose order is immaterial) form, say, a triple. The seven elements $a . . g$ arrange themselves into seven triples $A$. . G, viz.,

$$
\begin{array}{ll}
A=b c d & D=a d g \\
B=c a e & E=b e g \\
C=a b f & F=c f g
\end{array} \quad G=d e f
$$

This triple system $\Delta_{7}$ so set up on the elements $a \ldots g$ is invariant under a certain substitution-group $\Gamma_{\left(\Delta_{\gamma}\right)}^{\gamma}$. This $\Gamma_{\left(\Delta_{\gamma}\right)}^{\gamma}$ is exactly the $\Gamma^{\gamma}$ of holoedric transformation into itself of the $G_{8}$, since the $\Delta_{7}$ fully defines the product relations of the $G_{8}$, 
at least when we adjoin the system of equations, $a^{2}=1, \ldots g^{2}=1$, which is invariant under every substitution on the $a \ldots g$.

The $G_{8}$ and the $\Delta_{7}$ should be used together in studying the $\Gamma^{7}$. The extreme simplicity of the internal structure of the $G_{8}$ makes the properties of the $\Gamma^{\gamma}$ almost immediately evident. A substitution $\sigma$ of $\Gamma^{\gamma}$ is determined by the elements $a^{\prime}, b^{\prime}$, $c^{\prime}$ which it makes correspond to the generators $a, b, c$. Clearly any one of the seven elements may be chosen for $a^{\prime}$, any other one for $b^{\prime}$, and any one except the product $a^{\prime} b^{\prime}$ (i.e., except the third element of the triple containing $a^{\prime}, b^{\prime}$ ) for $c^{\prime}$. The $\Gamma^{7}$ has then the order $7.6 .4=168$. ${ }^{2}$ The $I_{168}^{7}$ is doubly transitive on the seven elements. I shall prove it simple and thereby identify it abstractly with the only existent simple* group of order 168 .

An enumeration of the substitutions $\sigma$ of the $\Gamma_{168}^{7}$ by period and by type $\nmid$ shows the following distribution :

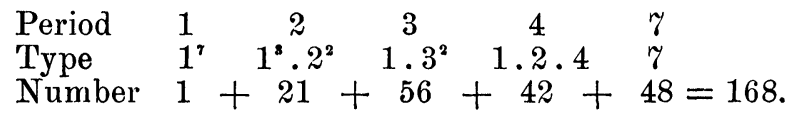

The $21 \sigma$ of period 2 are conjugate under the $I_{168}^{7}$; likewise the 56 of period 3 , and the 42 of period 4 . As to those of period 7 consider, say,

$$
\tau=(a b c f d g e)
$$

this $\tau$ does belong to $\Gamma_{168}^{7}$ for the triples

$$
a b f, b c c, c f g, f d e, d g a, g e b, e a c
$$

lie on its face cyclically and hence the triple system $\Delta_{\gamma}$ is invariant under $\tau$. Obviously the triples must lie cyclically on the face of every substitation $\sigma$ of period $\gamma$ of the $\Gamma_{168}^{7}$, not necessarily, however, exactly as in $\tau$; there are two and only two possibilities, both of which occur in the list of powers of $\tau$. In $\tau^{1}, \tau^{2}, \tau^{4}$ the triples lie as in $\tau$, in, say, the forward way; in $\tau^{3}, \tau^{5}, \tau^{\mathbf{6}}$ the triples lie as in

$$
\tau^{-1}=\tau^{6}=(a e g d f c b),
$$

in, say, the backward $\ddagger$ way. Thus the $48 \sigma$ of period 7 are half

* Holder: Die einfachen Gruppen im ersten und zweiten Hundert der Ordnungszahlen. Mathematische Annalen, vol. 40, pp. 55-88, 1892.

t The type of a substitution is the partition-symbol of its degree, arising from its complete expression in the cyclic notation. For instance, in the $\Gamma_{168}^{7} \sigma=(a b c g)(d f)$ has the type 1.2 .4 .

$\ddagger$ It will be noticed that the separation of the powers is according to the character of the exponent as a quadratic residue or non-residue of $\%$. 
of the forward and half of the backward style. All those of the same style are conjugate under the $\Gamma_{168}^{7}$. For let

$$
\tau_{1}=\left(t_{11} t_{12} \ldots t_{17}\right) \text { and } \tau_{2}=\left(\tau_{21} \tau_{22} \ldots \tau_{27}\right)
$$

be two of the same style; the triples lie on the faces of $\tau_{1}, \tau_{2}$ in the same relative positions; the substitution

$$
\lambda=\left(\begin{array}{lll}
t_{11} t_{12} & \ldots & t_{17} \\
t_{21} t_{22} & \ldots & t_{27}
\end{array}\right)
$$

transforms the triple system $\Delta_{7}$ into itself and hence belongs to $\Gamma_{168}^{7}$, and it obviously transforms $\tau_{1}$ into $\tau_{2}$. Equally clearly those of different style are not conjugate under the $\Gamma_{168}^{7}$. Nevertheless the eight cyclic groups of order $7 /$ are conjugate under the $\Gamma_{168}^{7}$.

Any self-conjugate sub-group $\Gamma^{7}$ of the $\Gamma_{168}^{7}$ must then contain besides the identity all or none of the substitutions $\sigma$ of each type of the list ( 7$)$. The fact that the diophantine equation

$$
1+21 \alpha+56 \beta+42 \gamma+48 \delta=d,
$$

where $\alpha, \beta, \gamma, \delta$ are respectively 0 or 1 and where $d$ is a divisor of 168 , has only the two solutions

$$
(\alpha, \beta, \gamma, \delta ; d)=(1,0,0,0 ; 1),(1,1,1,1 ; 168)
$$

shows that the $\Gamma_{168}^{7}$ is simple.* This identifies the group $\Gamma_{168}^{7}$; of which later.

The triple system $\Delta_{7}$ is given in (5) (6) from the standpoint of the seven elements $a \ldots g$. I give it again from the standpoint of the seven triples $A$. . G:

$$
\begin{array}{cll} 
& A & D \\
B & E \quad G \\
C & F
\end{array}
$$

* This method of proving the simplicity of a simple group when its elements and cyclic sub-groups have been classified as to conjugacy under the group was used by KLEIN for the icosuhedron $G_{B 0}$ (Vorlesungen über das Ikosaeder, p. 18, 1884) and has, us is well known, wide application. Is an instance known in which it does not apply? 
Obviously (14) (15) exhibit in the $A \ldots G$ a triple system $\Delta_{7}(A \ldots G)$ exactly * like the triple system $\Delta_{7}(\alpha \ldots g)$ exhibited by (5) (6).

Now a substitution $\sigma$ of $\Gamma_{168}^{7}$ permutes the $a \ldots g$, leaving $\Delta_{7}(a \ldots g)$ invariant; through the $a \ldots g$ it permutes the $A$. G $G$, but nevertheless leaves the $\Delta_{7}(A \ldots G)$ invariant. Call $\bar{\sigma}$ the substitution on the $A \ldots G$ which permutes the $A$.. G directly, just as $\sigma$ does indirectly. The totality of these substitutions $\bar{\sigma}$ constitutes a group, the group $\bar{\Gamma}_{168}^{7}$ of the $\Delta_{7}(A \ldots G)$, which is clearly on the one hand abstractly identical with the $\Gamma_{168}^{7}$ of the $\Delta_{\gamma}(a \ldots g)$ and on the other hand holoedrically isomorphic with the $\Gamma_{168}^{7}$ by the correspondence

$$
\sigma \sim \bar{\sigma}
$$

This holoedric isomorphism of the abstract $\Gamma_{168}^{7}$ with itself is not that arising from a transformation of the $\Gamma_{168}^{7}$ through one of its own elements. For such a transformation would change the substitution $\tau(8)$ of period $r$ into another one of the forward style, while (16) gives the correspondence

$$
\tau=(a b c f d g e) \sim \bar{\tau}=(A F G D E B C),
$$

where $\bar{\tau}$ is of the backward style.

The simple group $\Gamma_{168}$ (abstractly, there is but one) first appeared as the substitution-group $\Gamma_{168}^{8}$ connected with the modular equation for the transformation of elliptic functions of order \%. Galois discovered the existence of a resolvent of order $\%$ As to the development of the theory one may consult the introduction to a memoir of Mr. Gordan. $\dagger$

* A triple system $\Delta_{n}$ in $n$ elements can exist only if $n$ has the form $6 m+1$ or $6 m+3$, say the form $t$. All triple systems $\Delta_{3}$ are of the same class ; likewise for the $\Delta_{\gamma}, \Delta_{9}$. Triple systems $\Delta_{t}$ for every $t$ do exist, and indeed for every $t \geqq 13$ they fall into at least two distinct classes. As to these theorems these references:

NeTto : Zur Theorie der Tripelsysteme. Mathematische Annalen, vol. 42 , pp. $143-152,1893$.

Moore: Concerning Triple Systems. Mathematische Annalen, vol 43 , pp. 271-285, 1893.

JAN DE VRIEs: Zur Theorie der Tripelsysteme, Rendiconti del Circolo Matematico di Palermo, vol. 8, pp. 222-226, 1894.

† Gordas: Ueber Gleichungen siebenten Grades mit einer Gruppe von 168 Substitutionen. Mathematische Annalen, vol. 20, pp. 515-530, 1882. 
The triple system as determining invariant of the group $\Gamma_{168}^{7}$ was introduced by $\mathrm{Mr}$. Noether. ${ }^{*}$

The sub-groups of the group $\nmid \Gamma_{\frac{q\left(q^{2}-1\right)}{2}}^{q+1}$ connected $\ddagger$ with the modular equation for the transformation of prime order $q$ have been completely enumerated by Mr. Gierster, $\S$ thus illuminating Galois' theorem that the modular equation has resolvents of degree $q$ for $q=5,7,11$, but for no $q>11$,

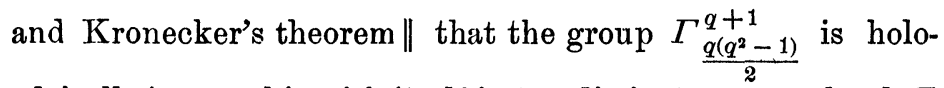
edrically isomorphic with itself in two distinct ways, and only 9 in those ways.

The idea** of the group $\Gamma^{n-1}$ of holoedric transformation into itself of a given group $G_{n}$ is, so far as I know, new. In the preceding sketch of the $\Gamma_{168}^{7}$ the perspicuous determination from the Abelian $G_{8}$ of the triple system $\Delta_{7}$ and of the $\Gamma_{168}^{7}$ is the only element of novelty.

The University of Chicago, November 5, 1894.

* Noetherr: Uber die Gleichungen achten Grades und ihr Auftreten in der Theorie der Curven vierter Ordnung. Mathematische Annalen, vol. 15, pp. 89-110, 1879).

t The $\Gamma_{\frac{q\left(q^{2}-1\right)}{2}}^{q+1}$ for $q>3$ is simple.

$\ddagger$ Connected with is of, if the numerical irrationality $\sqrt{(-1)^{\frac{q-1}{2}} \cdot q}$ is adjoined

\$GIERs'ter: Die Untergruppen der Galois'schen Gruppe der Modulargleichungen für den Fall eines primzahligen Transformationsgrades. Mathematische Annalen, vol. 18, pp. 319-365, 1881.

KRONECKER: Monatsberichte der Berliner Akademie, 1861.

ๆ As Gierster adds, loc. cit. p. 356.

** This concept and an application of it to the $\Gamma_{168}^{7}$ were communicated to the Mathematical Club of the University of Chicago in a paper presented January 19,1893, entitled "An existence-proof of the simple group of order 168 as a group of substitutions on 7 letters." 